\title{
Effect of hyperlipidemia on response to nonsurgical periodontal therapy: Statin users versus nonusers
}

\author{
Aditi Sangwan ${ }^{1}$, Shikha Tewari ${ }^{1}$, Harpreet Singh ${ }^{2}$, Rajinder Kumar Sharma ${ }^{1}$, \\ Satish Chander Narula ${ }^{1}$
}

Correspondence: Dr. Aditi Sangwan

Email: aditidalal86@yahoo.co.in

\begin{abstract}
'Department of Periodontics and Oral Implantology, Post Graduate Institute of Dental Sciences, Rohtak, Haryana, India,

2Department of Medicine, Post Graduate Institute of Medical Sciences, Rohtak, Haryana, India
\end{abstract}

\section{ABSTRACT}

Objective: To evaluate the response to nonsurgical periodontal therapy among hyperlipidemic subjects and whether statin use by hyperlipidemic subjects influences the response. Materials and Methods: This study was conducted on 107 chronic periodontitis subjects (35 normolipidemic [NL] controls, 36 hyperlipidemics on nonpharmacological therapy and 36 hyperlipidemics on statins). Periodontal (plaque index, gingival index [GI], probing depth [PD], and clinical attachment level [CAL]) and biochemical (plasma triglyceride [TG], total cholesterol [TC], low-density lipoprotein cholesterol [LDL-C], and high-DL-C [HDL-C] levels) examination was done at baseline and 3 months after nonsurgical periodontal treatment. Results: Both the NL and statin groups exhibited significantly greater improvement in GI as compared to the hyperlipidemic group on nonpharmacological therapy ( $P=0.004$ and 0.006 , respectively). Mean change in PD correlated negatively with baseline TC $(r=-0.306)$ and LDL-C $(r=-0.360)$ while mean change in GI positively correlated with baseline HDL-C $(r=0.219)$. Regression analyses revealed that mean change in PD was negatively associated with LDL-C $(\beta=-0.358, P<0.001)$ while mean change in GI was positively associated with HDL-C $(\beta=0.219, P=0.023)$. Conclusions: While higher baseline lipid levels were somewhat detrimental to the resolution of inflammation postperiodontal treatment, the inclusion of statin therapy among hyperlipidemic subjects seemed to improve clinical response as compared to those devoid of the drug. The findings of the study are suggestive of a possible adjunctive role of statins in periodontal treatment that warrants future studies.

Key words: Cholesterol, hydroxymethylglutaryl coenzyme A reductase inhibitors, hyperlipidemia, nonsurgical periodontal therapy, periodontitis

\section{INTRODUCTION}

Successful periodontal therapy chiefly comprises of procedures aimed at reducing the bacterial burden associated with dental plaque. This is most commonly achieved by scaling and root planing that constitutes the cornerstone of nonsurgical periodontal therapy. ${ }^{[1]}$ Various factors, both local as well as systemic, are known to affect the response to conventional periodontal treatment. ${ }^{[2-6]}$ A hyperlipidemic state could delay or hinder posttreatment periodontal healing since it has been reported to cause an up-regulation of pro-inflammatory cytokines such as tumor necrosis factor-alpha (TNF- $\alpha$ ) and interleukin-1 beta (IL-1 $\beta$ ) from monocytes/polymorpho nucleocytes (PMNs) as well as a reduction in growth factor production by macrophages. ${ }^{[7-9]}$ Hyperlipidemic subjects are cardiovascular disease (CVD) risk subjects and are frequently prescribed statins, which reduce serum cholesterol levels by causing competitive inhibition of 3-hydroxy-3-methylglutaryl coenzyme A reductase. ${ }^{[10,11]}$ Besides their lipid-lowering action, recent studies demonstrate that statins also cause reduced lipopolysaccharide-induced secretion of IL-6,

This is an open access article distributed under the terms of the Creative Commons Attribution-NonCommercial-ShareAlike 3.0 License, which allows others to remix, tweak, and build upon the work non-commercially, as long as the author is credited and the new creations are licensed under the identical terms.

For reprints contact: reprints@medknow.com

How to cite this article: Sangwan A, Tewari S, Singh H, Sharma RK, Narula SC. Effect of hyperlipidemia on response to nonsurgical periodontal therapy: Statin users versus nonusers. Eur J Dent 2016;10:69-76.

DOI: $10.4103 / 1305-7456.175685$ 
TNF- $\alpha$ and IL-1 $\beta$ by monocytes and macrophages, thus bringing about an anti-inflammatory action. ${ }^{[12,13]}$ On the basis of these properties, it could be hypothesized that statins might have a beneficial impact on periodontal health. This has been positively demonstrated by retrospective studies wherein the periodontal status of high-CVD risk subjects consuming statins was assessed. ${ }^{[14,15]}$ Furthermore, it was observed in a randomized controlled study that orally administered $20 \mathrm{mg}$ atorvastatin had beneficial effects in relation to alveolar bone loss and tooth mobility in chronic periodontitis. ${ }^{[16]}$ Consequently, a variation in response to periodontal treatment could also be expected on the basis of statin medication use prescribed for hyperlipidemia.

Therefore, the objective of this prospective study was to evaluate and compare the clinical response to nonsurgical periodontal treatment among hyperlipidemic subjects (divided on the basis of statin use) with normolipidemic (NL) controls. To the best of our knowledge, this is the first study conducted with this specific aim.

\section{MATERIALS AND METHODS}

The current study was designed as a prospective, comparative, and single-blind clinical study with a follow-up period of 3 months. It was carried out in the Department of Periodontics and Oral Implantology in alliance with the Department of Medicine. The study protocol was constructed keeping in consideration the Declaration of Helsinki, and ethical approval (PGBOS/ UHSR/Perio/04/2010/Dated 18.2.2010) was obtained from the Institutional Review Board. Subjects were enrolled from January 2011 to October 2011 among those who attended the regular outpatient clinic of the Department of Medicine.

Criteria adopted for inclusion in the study were: Adults having chronic periodontitis with $\geq 16$ natural teeth excluding third molars and no history of periodontal treatment within past 6 months. The defining factor for the diagnosis of chronic periodontitis was adopted from Page and Eke (2007): $\geq 2$ interproximal sites with clinical attachment level (CAL) $\geq 4 \mathrm{~mm}$ (not on the same tooth) or $\geq 2$ interproximal sites with probing depth (PD) $\geq 5 \mathrm{~mm}$ (not on the same tooth) ${ }^{[17]}$ Care was taken during subject enlisting to establish uniformity of study cohort regarding baseline periodontal parameters.

The following conditions made the case valid for exclusion: History of systemic disease capable of affecting lipid metabolism or progression of periodontal disease such as diabetes mellitus, impaired glucose tolerance or other endocrine diseases, CVD, rheumatic disease, chronic renal failure, nephrotic syndrome; pregnancy, lactation or hormone replacement therapy within duration of study, chronic treatment with nonsteroidal anti-inflammatory drugs, bisphosphonates treatment, glucocorticoid treatment, or use of any other drug known to affect bone calcium metabolism, and systemic infection or any history of systemic antibiotic therapy within 3 months preceding the study. Smokers and ex-smokers were also not included. An oral questionnaire was utilized to gather data regarding gender, age, smoking status, and medication use if any. To confirm strict adherence to enrollment criteria, a detailed medical history was recorded in conjunction with referring physician's consultation, whenever required. For the purpose of calculation of body mass index (BMI) as $\left(\mathrm{kg} / \mathrm{m}^{2}\right)$, anthropometric measurements (weight $[\mathrm{kg}]$ ) and height $[\mathrm{m}]$ ) were recorded.

A total of 446 subjects were appraised, out of which 112 subjects were found suitable for inclusion. The study participants were distributed into three groups on basis of serum lipid levels and treatment strategies-control group: NL subjects, test group 1: Hyperlipidemic subjects on nonpharmacological therapy comprising of diet and exercise for duration $\geq 3$ months (HL-N), and test group 2: Hyperlipidemic subjects taking statin medication (atorvastatin [20 mg/day]) for duration $\geq 3$ months (HL-S). Informed consent was obtained from each patient at outset of the study.

\section{Periodontal examination}

Assessment of each tooth was carried out at four sites (mesiobuccal, midbuccal, distobuccal, and oral) and periodontal parameters recorded included plaque index $(\mathrm{PI}),^{[18]}$ gingival index $(\mathrm{GI}){ }^{[19]} \mathrm{CAL}$ and PD using Williams periodontal probe (Hu-Friedy).

Investigator blinding was ascertained, and interexaminer variability was prevented by involving only one investigator for recruitment of participants into different groups while another one handled the oral examination and periodontal treatment. To determine examiner reproducibility, clinical periodontal data recording on ten patients was done twice followed by calculation of the percentage of the sites examined where the scores were identical or within $\pm 1 \mathrm{~mm}$. Assessment of the mean difference in the scores (with 90\% accuracy) demonstrated that there was no systematic bias in the measurements. 


\section{Biochemical assessment for serum lipids}

Venous blood samples were collected from an antecubital vein after an overnight fasting interval for the measurement of total cholesterol (TC), plasma triglyceride (TG), low-density lipoprotein cholesterol (LDL-C), and high-DL-C (HDL-C) levels with the aid of autoanalyzer (Model Erba XL-300, Mannheim). For the purpose of diagnosing subjects as hyperlipidemic, the cut-off points were: TC: $>200$ mg/dl, TG: $>200 \mathrm{mg} / \mathrm{dl}$, LDL-C: $>130 \mathrm{mg} / \mathrm{dl}$, and HDL-C: $<35 \mathrm{mg} / \mathrm{dl}$.

\section{Periodontal treatment}

Nonsurgical periodontal treatment, comprising oral hygiene instructions and scaling and root planing with both ultrasonic instrumentation (Satelec Suprasson P5 booster) and Gracey curettes (Hu-Friedey) was provided to all participants. It was ensured that treatment was completed within 2 weeks in up to a maximum of four sittings. Only mechanical oral hygiene aids such as toothbrushes and floss were allowed to be used by participants and use of mouthwashes or antibiotics was prohibited during the study duration. Reexamination of participants (both periodontal and biochemical) was conducted according to the baseline specifications after 3 months.

\section{Statistical analysis}

The sample size was calculated using statistical analysis (Gpower version 2.0, Bonn, Germany). A power calculation indicated that when the sample size was 102 , the test would have $>90 \%$ power at an effect size of 0.40 , assuming a significance level of $5 \%$.

All statistical analyses were carried out utilizing statistical software (SPSS, v.15.0 for Windows, SPSS, Chicago, IL.) with a two-tailed $P=0.05$ specified as a threshold for significance. Data were illustrated using means and standard deviation. The normality of distribution of data was investigated using the Kolmogorov-Smirnov test. Kruskal-Wallis followed by the Mann-Whitney U-test was used to analyze differences among groups. The Chi-square test was employed to test for significant differences among groups with respect to gender. Comparison of periodontal and biochemical parameters at baseline and 3 months after periodontal treatment was done using Wilcoxon signed-rank test. Partial correlations between baseline lipid levels and mean change in periodontal parameters were evaluated after controlling for confounders (gender, age, and
BMI). Multiple stepwise regression analyses were carried out to examine the association between lipid parameters and improvement observed in periodontal parameters after treatment.

\section{RESULTS}

Of the 112 subjects enrolled, 5 subjects dropped out and 107 subjects reported for 3 months follow-up control group: NL subjects (NL) $(n=35)$, test group 1: Hyperlipidemic subjects on nonpharmacological therapy comprising of diet and exercise for duration $\geq 3$ months (HL-N) $(n=36)$, and test group 2: Hyperlipidemic subjects taking statin medication (atorvastatin [20 mg/day]) for duration $\geq 3$ months (HL-S) $(n=36)$.

The baseline characteristics of the subjects are shown in Table 1. At baseline, there were no significant differences in age, gender, number of teeth, PI, GI, PD, and CAL among the groups while BMI significantly differed only between control and statin group. No change in medication during the study period was reported in the HL-S group.

In all the groups, nonsurgical periodontal therapy resulted in a significant decrease $(P<0.001)$ in mean PI, GI, PD, and CAL after 3 months [Table 2]. There was no difference in response to periodontal treatment between the three groups regarding PD, CAL, and PI. In the case of GI, both NL group and HL-S group exhibited statistically significantly greater improvement as compared to the HL-N group $(P=0.004$ and 0.006 , respectively) [Table 3]. There was no significant difference in mean change in GI between NL group and HL-S group. Biochemical examination at the 3 months follow-up revealed that lipid parameters across all three groups were reduced (as compared to the baseline), but this change was not significant for any of the lipid fractions [Table 2].

Table 4 shows a correlation between baseline lipid levels and mean change in periodontal parameters after controlling for confounders (age, gender, and BMI). Mean change in PD was found to be negatively and significantly correlated with baseline TC and LDL-C levels (correlation coefficient $=-0.306$ and -0.360 , respectively) while mean change in GI was positively correlated with baseline HDL-C levels (correlation coefficient $=0.219$ ). Results of multiple regression analysis revealed that mean posttreatment gain in PD was negatively associated with baseline LDL-C $(\beta=-0.358, P=<0.001)$ while mean change in GI 
Sangwan, et al.: Serum lipids, statins and periodontal therapy

\begin{tabular}{|c|c|c|c|c|}
\hline & NL $(n=35)$ & HL-N $(n=36)$ & HL-S $(n=36)$ & Total $(n=107)$ \\
\hline Age (years) & $43.00 \pm 10.73$ & $42.06 \pm 9.93$ & $44.56 \pm 10.44$ & $43.21 \pm 10.32$ \\
\hline Male/female & $17 / 18$ & $19 / 17$ & $24 / 12$ & $60 / 47$ \\
\hline TG (mg/dl) & $133.03 \pm 27.86$ & $253.33 \pm 67.62$ & $220.89 \pm 73.99$ & $203.07 \pm 78.44$ \\
\hline $\mathrm{TC}(\mathrm{mg} / \mathrm{dl})$ & $139.23 \pm 25.86$ & $233.97 \pm 38.22$ & $218.06 \pm 19.47$ & $197.63 \pm 50.38$ \\
\hline $\mathrm{HDL}(\mathrm{mg} / \mathrm{dl})$ & $43.60 \pm 5.60$ & $40.97 \pm 7.19$ & $46.47 \pm 6.12$ & $43.68 \pm 6.68$ \\
\hline LDL (mg/dl) & $68.94 \pm 25.06$ & $142.36 \pm 37.37$ & $127.47 \pm 24.56$ & $113.34 \pm 43.20$ \\
\hline BMI $\left(\mathrm{kg} / \mathrm{m}^{2}\right)$ & $23.69 \pm 2.93$ & $24.94 \pm 2.58$ & $26.43 \pm 3.95$ & $25.03 \pm 3.37$ \\
\hline $\mathrm{PD}(\mathrm{mm})$ & $2.93 \pm 0.46$ & $3.05 \pm 0.39$ & $3.11 \pm 0.62$ & $3.03 \pm 0.50$ \\
\hline $\mathrm{CAL}(\mathrm{mm})$ & $3.50 \pm 0.70$ & $3.55 \pm 0.64$ & $3.99 \pm 1.07$ & $3.68 \pm 0.84$ \\
\hline $\mathrm{GI}$ & $1.44 \pm 0.28$ & $1.56 \pm 0.31$ & $1.47 \pm 0.31$ & $1.49 \pm 0.30$ \\
\hline $\mathrm{PI}$ & $1.33 \pm 0.27$ & $1.42 \pm 0.34$ & $1.35 \pm 0.37$ & $1.36 \pm 0.33$ \\
\hline Number of teeth & $26.51 \pm 1.69$ & $25.61 \pm 2.63$ & $25.33 \pm 2.74$ & $25.81 \pm 2.44$ \\
\hline
\end{tabular}

NL: Normolipidemic, HL-N: Hyperlipidemic group on nonpharmacological therapy, HL-S: Hyperlipidemic subjects on statins, TG: Triglyceride, PD: Probing depth, LDL: Low-density lipoprotein, HDL: High-density lipoprotein, TC: Total cholesterol, PI: Plaque index, GI: Gingival index, CAL: Clinical attachment level, BMI: Body mass index

Table 2: Comparison of lipid and periodontal parameters at baseline and 3 months after periodontal treatment

\begin{tabular}{|c|c|c|c|c|c|c|c|c|c|}
\hline & \multicolumn{3}{|c|}{ NL group } & \multicolumn{3}{|c|}{ HL-N group } & \multicolumn{3}{|c|}{ HL-S group } \\
\hline & Baseline & Posttreatment & $\begin{array}{l}\text { Significant } \\
(P)\end{array}$ & Baseline & Posttreatment & $\begin{array}{l}\text { Significant } \\
(P)\end{array}$ & Baseline & Posttreatment & $\begin{array}{l}\text { Significant } \\
(P)\end{array}$ \\
\hline \multicolumn{10}{|l|}{$\begin{array}{l}\text { Lipid } \\
\text { parameters }\end{array}$} \\
\hline TG (mg/dl) & $133.03 \pm 27.86$ & $131.20 \pm 26.03$ & NS & $253.33 \pm 67.62$ & $251.03 \pm 65.03$ & NS & $220.89 \pm 73.99$ & $219.47 \pm 68.65$ & NS \\
\hline $\mathrm{TC}(\mathrm{mg} / \mathrm{dl})$ & $139.23 \pm 25.86$ & $137.49 \pm 28.17$ & NS & $233.97 \pm 38.22$ & $231.92 \pm 38.13$ & NS & $218.06 \pm 19.47$ & $214.89 \pm 19.12$ & NS \\
\hline $\mathrm{HDL}(\mathrm{mg} / \mathrm{dl})$ & $43.60 \pm 5.60$ & $43.31 \pm 5.38$ & NS & $40.97 \pm 7.19$ & $40.06 \pm 7.37$ & NS & $46.47 \pm 6.12$ & $46.00 \pm 7.29$ & NS \\
\hline LDL (mg/dl) & $68.94 \pm 25.06$ & $67.97 \pm 28.12$ & NS & $142.36 \pm 37.37$ & $141.67 \pm 36.24$ & NS & $127.47 \pm 24.56$ & $124.94 \pm 24.52$ & NS \\
\hline \multicolumn{10}{|c|}{$\begin{array}{l}\text { Periodontal } \\
\text { parameters }\end{array}$} \\
\hline $\mathrm{PD}(\mathrm{mm})$ & $2.93 \pm 0.46$ & $2.27 \pm 0.54$ & $<0.001$ & $3.05 \pm 0.39$ & $2.42 \pm 0.52$ & $<0.001$ & $3.11 \pm 0.62$ & $2.46 \pm 0.68$ & $<0.001$ \\
\hline $\mathrm{CAL}(\mathrm{mm})$ & $3.50 \pm 0.69$ & $3.14 \pm 0.71$ & $<0.001$ & $3.55 \pm 0.64$ & $3.22 \pm 0.60$ & $<0.001$ & $3.99 \pm 1.07$ & $3.63 \pm 1.07$ & $<0.001$ \\
\hline $\mathrm{GI}$ & $1.44 \pm 0.28$ & $0.79 \pm 0.21$ & $<0.001$ & $1.56 \pm 0.31$ & $1.00 \pm 0.32$ & $<0.001$ & $1.47 \pm 0.31$ & $0.84 \pm 0.31$ & $<0.001$ \\
\hline $\mathrm{PI}$ & $1.33 \pm 0.27$ & $0.90 \pm 0.18$ & $<0.001$ & $1.42 \pm 0.34$ & $0.95 \pm 0.30$ & $<0.001$ & $1.35 \pm 0.37$ & $0.91 \pm 0.21$ & $<0.001$ \\
\hline
\end{tabular}

NS: Nonsignificant, NL: Normolipidemic, HL-N: Hyperlipidemic group on nonpharmacological therapy, HL-S: Hyperlipidemic subjects on statins, TG: Triglyceride, PD: Probing depth, LDL: Low-density lipoprotein, HDL: High-density lipoprotein, TC: Total cholesterol, PI: Plaque index, GI: Gingival index, CAL: Clinical attachment level

\begin{tabular}{|c|c|c|c|c|}
\hline & PD & CAL & GI & PI \\
\hline NL versus HL-N & 0.059 & 0.062 & $0.004^{*}$ & 0.300 \\
\hline NL versus HL-S & 0.447 & 0.616 & 0.831 & 0.259 \\
\hline HL-N versus HL-S & 0.103 & 0.147 & $0.006^{*}$ & 0.058 \\
\hline \multicolumn{5}{|c|}{$\begin{array}{l}\text { *Significant difference at } 0.05 \text { level. NL: Normolipidemic, HL-N: Hyperlipidemic } \\
\text { group on nonpharmacological therapy, HL-S: Hyperlipidemic subjects on } \\
\text { statins, PI: Plaque index, GI: Gingival index, CAL: Clinical attachment level, } \\
\text { PD: Probing depth }\end{array}$} \\
\hline
\end{tabular}

was positively associated with baseline HDL-C levels $(\beta=0.219, P=0.023)$.

\section{DISCUSSION}

Our study aimed to assess the response to nonsurgical periodontal treatment among hyperlipidemic subjects while also comparing the same between statin users and nonusers. The comparison was based on clinical parameters comprising of CAL, PD, GI, and PI. Both the NL and statin groups exhibited significantly greater improvement in GI ( $P=0.004$ and 0.006 , respectively) as compared to HL-N, thereby leading to rejection of the null hypothesis. In this study, the mean changes in periodontal parameters were found correlated with baseline lipid values after controlling for potential confounders (age, gender, and BMI) [Table 4]. The findings of regression analyses also indicated that clinical response to periodontal treatment is weakened by high serum LDL-C while high HDL-C favors resolution of periodontal inflammation following therapy.

Periodontitis is a type of inflammatory tissue wound caused by constant microbiologic injury and is conventionally managed by mechanical removal of supra- and sub-gingival plaque that leads to resolution of inflammation and thereby 
Sangwan, et al.: Serum lipids, statins and periodontal therapy

\begin{tabular}{|c|c|c|c|c|c|c|c|}
\hline & $\triangle \mathrm{CAL}$ & $\Delta \mathbf{G I}$ & $\Delta \mathrm{PI}$ & TG & TC & HDL & LDL \\
\hline \multicolumn{8}{|l|}{$\Delta \mathrm{PD}$} \\
\hline Correlation & -0.062 & -0.159 & -0.066 & -0.017 & $-0.306^{*}$ & 0.073 & $-0.360^{*}$ \\
\hline Significance (two-tailed) & 0.533 & 0.106 & 0.506 & 0.867 & 0.002 & 0.464 & $<0.001$ \\
\hline \multicolumn{8}{|l|}{$\Delta \mathrm{CAL}$} \\
\hline Correlation & $x$ & 0.114 & 0.107 & 0.088 & 0.010 & 0.028 & -0.026 \\
\hline Significance (two-tailed) & & 0.249 & 0.280 & 0.377 & 0.919 & 0.776 & 0.793 \\
\hline \multicolumn{8}{|l|}{$\Delta \mathrm{Gl}$} \\
\hline Correlation & & $x$ & -0.030 & -0.057 & -0.070 & $0.219^{*}$ & -0.094 \\
\hline Significance (two-tailed) & & & 0.765 & 0.564 & 0.481 & 0.026 & 0.345 \\
\hline \multicolumn{8}{|l|}{$\Delta \mathrm{PI}$} \\
\hline Correlation & & & $x$ & 0.010 & 0.153 & 0.049 & 0.167 \\
\hline Significance (two-tailed) & & & & 0.921 & 0.121 & 0.619 & 0.091 \\
\hline \multicolumn{8}{|l|}{ TG } \\
\hline Correlation & & & & $x$ & $0.594^{*}$ & -0.073 & $0.335^{*}$ \\
\hline Significance (two-tailed) & & & & & $<0.001$ & 0.462 & 0.001 \\
\hline \multicolumn{8}{|l|}{ TC } \\
\hline Correlation & & & & & $x$ & -0.028 & $0.946^{*}$ \\
\hline Significance (two-tailed) & & & & & & 0.779 & $<0.001$ \\
\hline \multicolumn{8}{|l|}{$\mathrm{HDL}$} \\
\hline Correlation & & & & & & $x$ & -0.163 \\
\hline Significance (two-tailed) & & & & & & & 0.098 \\
\hline \multicolumn{8}{|l|}{ LDL } \\
\hline Correlation & & & & & & & $x$ \\
\hline Significance (two-tailed) & & & & & & & \\
\hline
\end{tabular}

promoting clinical healing and repair. ${ }^{[1]}$ Proper wound healing depends on various cytokines and growth factors of which the macrophages are a primary source. This release of wound repair aiding factors (such as platelet-derived growth factor and transforming growth factor beta 1 [TGF- $\beta$ 1]) from macrophages has been established to be reduced by serum lipids, ${ }^{[9]}$ thereby strengthening the premise that elevated lipid levels contribute to delayed wound healing. Furthernore, high serum lipids are reportedly associated with an enhanced production of pro-inflammatory cytokines (TNF- $\alpha$ and IL-1 $\beta$ ) from monocytes/PMNs, ${ }^{[7,8]}$ thus propagating a pro-inflammatory state. Consequently, alterations in immune cell function caused by hyperlipidemia could delay or hamper posttreatment periodontal healing and provide a biologic explanation for the observed association between high serum lipids and comparatively poor response to periodontal therapy. It is also interesting to note that higher values of baseline HDL-C were found associated positively with mean posttreatment change in GI. An explanation for this observation, most likely involves several biological effects pertaining to the antioxidant and anti-inflammatory activities of HDL-C. ${ }^{[20]}$
In a previous cross-sectional study, hyperlipidemic subjects were found to have a periodontal status poorer than NLs while the periodontal status of statin users did not differ significantly from that of NLs. ${ }^{[21]}$ The current study revealed that the HL-S, as well as NL controls, experienced a significantly higher reduction in GI postperiodontal therapy as compared to the hyperlipidemic nonstatin users. This could indicate that hyperlipidemics (without statins) were relatively less responsive to periodontal treatment. In addition, statistically, similar reduction in GI was observed in statin users and normolipidmics, despite higher lipid parameters in former. The beneficial effects of statins on the periodontium have been previously investigated. In the retrospective cohort study by Cunha-Cruz et al., ${ }^{[14]}$ it was reported that statin consumption in the 3 years interval after initial periodontal evaluation resulted in significantly decreased the risk of tooth loss. Moreover, a study conducted among Finnish subjects by Lindy et al..$^{[15]}$ also corroborated our hypothesis. According to the results of their research, when the periodontal status of statin users was retrospectively compared with that of nonstatin users, the former were found to have lesser periodontal inflammation. A recent randomized 
controlled trial contributed major evidence in favour of statins having a positive role in periodontal management. ${ }^{[16]}$ They found orally administered $20 \mathrm{mg}$ atorvastatin to be effective regarding alveolar bone loss and tooth mobility in chronic periodontitis subjects.

Both the above-mentioned findings of this study could be attributed to certain beneficial actions of statins. It has been found that statins possess a potentially anti-inflammatory action by virtue of their ability to reduce secretion of pro-inflammatory molecules such as matrix metalloproteinases, ${ }^{[22]}$ C-reactive proteins and TNF- $\alpha^{[23]}$ and inhibition of IL-6. ${ }^{[24]}$ Destructive processes in periodontal inflammation have been found associated with the aforementioned pro-inflammatory molecules ${ }^{[25,26]}$ while resolution following periodontal intervention has been found linked to diminished levels of inflammatory cytokines. ${ }^{[27]}$ Therefore, it is possible that a direct anti-inflammatory action of statins could be adjunctive in promoting periodontal healing following nonsurgical therapy. This assumption in the current context is also supported by recent studies that suggest that oral administration of clinical doses of statins could have a local anti-inflammatory action on oral tissues. ${ }^{[28,29]}$ In a study conducted to evaluate the effects of periodontal treatment on serum and gingival crevicular fluid (gcf) levels of pro-inflammatory cytokines, hyperlipidemic subjects were divided on the basis of antilipemic therapy like the current study. ${ }^{[30]}$ It was found that the hyperlipidemic group on statins displayed a significant decrease in gcf IL-6 as compared to the other two groups, thereby supporting our hypothesis. On similar lines, another study compared the IL-1 $\beta$ levels in gcf among chronic periodontitis patients with and without statin therapy. ${ }^{[31]}$ They found the titers of gcf IL-1 $\beta$ higher in the group not consuming statins, thereby supporting the anti-inflammatory role of statins for periodontal tissues. A recent research observed that hyperlipidemic patients on statin treatment had higher levels of the bone protective biomarker osteoprotegerin as compared to hyperlipidemic nonstatin users. ${ }^{[32]}$ In the same study, statin users also displayed a mean decrease of $0.8 \mathrm{~mm}$ in CAL as compared to NL control subjects. In the retrospective cohort study by Cunha-Cruz et al., ${ }^{[14]}$ it was reported that statin consumption in the 3 years interval after initial periodontal evaluation resulted in significantly decreased the risk of tooth loss. Moreover, a study conducted among finnish subjects by Lindy et al..$^{[15]}$ also corroborated our hypothesis. According to the results of their research, when the periodontal status of statin users was retrospectively compared with that of nonstatin users, the former were found to have lesser periodontal inflammation. A recent randomized controlled trial ${ }^{[16]}$ contributed major evidence in favour of statins having a positive role in periodontal management. They found orally administered $20 \mathrm{mg}$ atorvastatin to be effective regarding alveolar bone loss and tooth mobility in chronic periodontitis subjects. Lastly, a recent research conducted to determine the minimum inhibitory concentration of simvastatin for periodontal pathogens successfully demonstrated the anti-microbial action of statins and thus added a fresh supportive perspective to our arguments. ${ }^{[33]}$

Another mechanism that could explain the favourable effect of statins on the periodontal tissues could be that reduced serum lipid levels as a result of statin use itself could benefit periodontal tissues indirectly since higher lipid levels have been found associated with relatively poor periodontal status. ${ }^{[34,35]}$

Although both the above-mentioned mechanisms could have synergistically produced the observed effects, the direct interpretation of the data obtained from the present study points towards the lipid-lowering action of statins as the main mechanism since the serum lipid levels of HL-S group were significantly lower than those of HL-N group $(P<0.05)$. Furthermore, the mean changes in periodontal parameters (PD) were found to be negatively correlated with baseline total and LDL-C levels [Table 4]. However, this alone seems insufficient at explaining as to how the postperiodontal treatment response of HL-S was similar to NL group even though it had much higher baseline lipid levels [Tables 1 and 3]. Another observation of the current study was that despite the BMI of HL-S group being significantly higher than that of the HL-N group, both groups responded in a similar manner to the periodontal treatment. Since it has been reported by many recent studies $^{[36,37]}$ that BMI and periodontal disease are positively associated in a way such that higher BMI could aggravate periodontal disease expression by increasing the level of inflammatory cytokines in circulation, ${ }^{[38]}$ this finding calls for an alternative explanation. The reasoning for this finding repeatedly lures us into considering the direct anti-inflammatory role of statins on oral tissues. While there is a distinct possibility that this assumption holds true, we cannot assertively suggest the same since measurement of inflammatory markers was not carried out in this 
study to substantiate such claims. This was, in fact, a limitation of the current study.

Finally, the strengths of the current study deserve a mention. A group of NL subjects was included to serve as control group. Care was taken to ensure that all relevant baseline periodontal parameters did not differ significantly among the three study groups to facilitate a fair comparison of posttreatment healing. The restriction of participation of smokers as well as of individuals having any known risk factors for periodontitis and/or hyperlipidemia was also done to ensure homogeneity of study subjects.

To conclude, data from the current study revealed that 3 months after periodontal treatment, hyperlipidemic subjects without statins had more gingival inflammation as compared to the other subjects while the periodontal status of hyperlipidemic statin users was similar to NL subjects. This further strengthens the hypothesized link between lipid metabolic disturbances and periodontal disease while also suggesting a potential adjunctive role of statins in periodontal treatment strategies. It is imperative that large-scale randomized studies designed to overcome the limitations of the present study are conducted to confirm these findings and gather more evidence for the development of novel periodontal treatment options.

\section{Financial support and sponsorship Nil.}

\section{Conflicts of interest}

There are no conflicts of interest.

\section{REFERENCES}

1. Drisko $\mathrm{CH}$. Nonsurgical periodontal therapy. Periodontol 2000 2001;25:77-88.

2. Tervonen T, Karjalainen K. Periodontal disease related to diabetic status. A pilot study of the response to periodontal therapy in type 1 diabetes. J Clin Periodontol 1997;24:505-10.

3. Wan CP, Leung WK, Wong MC, Wong RM, Wan P, Lo EC, et al. Effects of smoking on healing response to non-surgical periodontal therapy: A multilevel modelling analysis. J Clin Periodontol 2009;36:229-39.

4. Matthews DC. Periodontal medicine: A new paradigm. J Can Dent Assoc 2000;66:488-91.

5. Bertoldi C, Lalla M, Pradelli JM, Cortellini P, Lucchi A, Zaffe D. Risk factors and socioeconomic condition effects on periodontal and dental health: A pilot study among adults over fifty years of age. Eur J Dent 2013;7:336-46.

6. Sood P, Narang R, Swathi V, Mittal L, Jha K, Gupta A. Dental patient's knowledge and perceptions about the effects of smoking and role of dentists in smoking cessation activities. Eur J Dent 2014;8:216-23

7. Niemann-Jönsson A, Dimayuga P, Jovinge S, Calara F, Ares MP, Fredrikson GN, et al. Accumulation of LDL in rat arteries is associated with activation of tumor necrosis factor-alpha expression. Arterioscler Thromb Vasc Biol 2000;20:2205-11.
8. Thomas CE, Jackson RL, Ohlweiler DF, Ku G. Multiple lipid oxidation products in low density lipoproteins induce interleukin-1 beta release from human blood mononuclear cells. J Lipid Res 1994;35:417-27.

9. Chu X, Newman J, Park B, Nares S, Ordonez G, Iacopino AM. In vitro alteration of macrophage phenotype and function by serum lipids. Cell Tissue Res 1999;296:331-7.

10. Endo A, Kuroda M, Tanzawa K. Competitive inhibition of 3-hydroxy-3-methylglutaryl coenzyme A reductase by ML-236A and ML-236B fungal metabolites, having hypocholesterolemic activity. FEBS Lett 1976;72:323-6.

11. Shepherd J, Cobbe SM, Ford I, Isles CG, Lorimer AR, MacFarlane PW, et al. Prevention of coronary heart disease with pravastatin in men with hypercholesterolemia. West of Scotland Coronary Prevention Study Group. N Engl J Med 1995;333:1301-7.

12. Ikeda U, Shimada K. Statins and monocytes. Lancet 1999;353:2070.

13. Rosenson RS, Tangney CC, Casey LC. Inhibition of proinflammatory cytokine production by pravastatin. Lancet 1999;353:983-4.

14. Cunha-Cruz J, Saver B, Maupome G, Hujoel PP. Statin use and tooth loss in chronic periodontitis patients. J Periodontol 2006;77:1061-6.

15. Lindy O, Suomalainen K, Mäkelä M, Lindy S. Statin use is associated with fewer periodontal lesions: A retrospective study. BMC Oral Health 2008;8:16.

16. Fajardo ME, Rocha ML, Sánchez-Marin FJ, Espinosa-Chávez EJ. Effect of atorvastatin on chronic periodontitis: A randomized pilot study. J Clin Periodontol 2010;37:1016-22.

17. Page RC, Eke PI. Case definitions for use in population-based surveillance of periodontitis. J Periodontol 2007;78 7 Suppl: 1387-99.

18. Silness J, Loe H. Periodontal disease in pregnancy. II. Correlation between oral hygiene and periodontal condtion. Acta Odontol Scand 1964;22:121-35.

19. Loe H, Silness J. Periodontal disease in pregnancy. I. Prevalence and severity. Acta Odontol Scand 1963;21:533-51.

20. Podrez EA. Anti-oxidant properties of high-density lipoprotein and atherosclerosis. Clin Exp Pharmacol Physiol 2010;37:719-25.

21. Sangwan A, Tewari S, Singh H, Sharma RK, Narula SC. Periodontal status and hyperlipidemia: Statin users versus non-users. J Periodontol 2013;84:3-12.

22. Wong B, Lumma WC, Smith AM, Sisko JT, Wright SD, Cai TQ. Statins suppress THP-1 cell migration and secretion of matrix metalloproteinase 9 by inhibiting geranylgeranylation. J Leukoc Biol 2001;69:959-62

23. Koh KK, Son JW, Ahn JY, Jin DK, Kim HS, Choi YM, et al. Comparative effects of diet and statin on NO bioactivity and matrix metalloproteinases in hypercholesterolemic patients with coronary artery disease. Arterioscler Thromb Vasc Biol 2002;22:e19-23.

24. Undas A, Brozek J, Musial J. Anti-inflammatory and antithrombotic effects of statins in the management of coronary artery disease. Clin Lab 2002;48:287-96.

25. Offenbacher S. Periodontal diseases: Pathogenesis. Ann Periodontol 1996;1:821-78.

26. Noack B, Genco RJ, Trevisan M, Grossi S, Zambon JJ, De Nardin E. Periodontal infections contribute to elevated systemic C-reactive protein level. J Periodontol 2001;72:1221-7.

27. Sun WL, Chen LL, Zhang SZ, Ren YZ, Qin GM. Changes of adiponectin and inflammatory cytokines after periodontal intervention in type 2 diabetes patients with periodontitis. Arch Oral Biol 2010;55:970-4.

28. Yang H, Feng Y, Luan Y. Determination of simvastatin in human plasma by liquid chromatography-mass spectrometry. J Chromatogr B Analyt Technol Biomed Life Sci 2003;785:369-75.

29. Sakoda K, Yamamoto M, Negishi Y, Liao JK, Node K, Izumi Y. Simvastatin decreases IL-6 and IL-8 production in epithelial cells. J Dent Res 2006;85:520-3.

30. Fentoglu O, Kirzioglu FY, Ozdem M, Koçak H, Sütçü R, Sert T. Proinflammatory cytokine levels in hyperlipidemic patients with periodontitis after periodontal treatment. Oral Dis 2012;18:299-306.

31. Suresh S, Narayana S, Jayakumar P, Sudhakar U, Pramod V. Evaluation of anti-inflammatory effect of statins in chronic periodontitis. Indian J Pharmacol 2013;45:391-4.

32. Magán-Fernández A, Papay-Ramírez L, Tomás J, Marfil-Álvarez R, Rizzo M, Bravo M, et al. Association of simvastatin and hyperlipidemia with periodontal status and bone metabolism markers. J Periodontol 2014;85:1408-15.

33. Emani S, Gunjiganur GV, Mehta DS. Determination of the antibacterial activity of simvastatin against periodontal pathogens, Porphyromonas 
gingivalis and Aggregatibacter actinomycetemcomitans: An in vitro study. Contemp Clin Dent 2014;5:377-82.

34. Fentoglu O, Oz G, Tasdelen P, Uskun E, Aykaç Y, Bozkurt FY. Periodontal status in subjects with hyperlipidemia. J Periodontol 2009;80:267-73.

35. Awartani F, Atassi F. Evaluation of periodontal status in subjects with hyperlipidemia. J Contemp Dent Pract 2010;11:033-40.

36. Ekuni D, Yamamoto T, Koyama R, Tsuneishi M, Naito K, Tobe K. Relationship between body mass index and periodontitis in young Japanese adults. J Periodontal Res 2008;43:417-21.

37. Pataro AL, Costa FO, Cortelli SC, Cortelli JR, Abreu MH, Costa JE. Association between severity of body mass index and periodontal condition in women. Clin Oral Investig 2012;16:727-34.

38. Dandona P, Aljada A, Bandyopadhyay A. Inflammation: The link between insulin resistance, obesity and diabetes. Trends Immunol 2004;25:4-7.

\begin{tabular}{|l|l|}
\hline \multicolumn{2}{|c|}{ Access this article online } \\
\hline Quick Response Code: & \\
\hline
\end{tabular}

